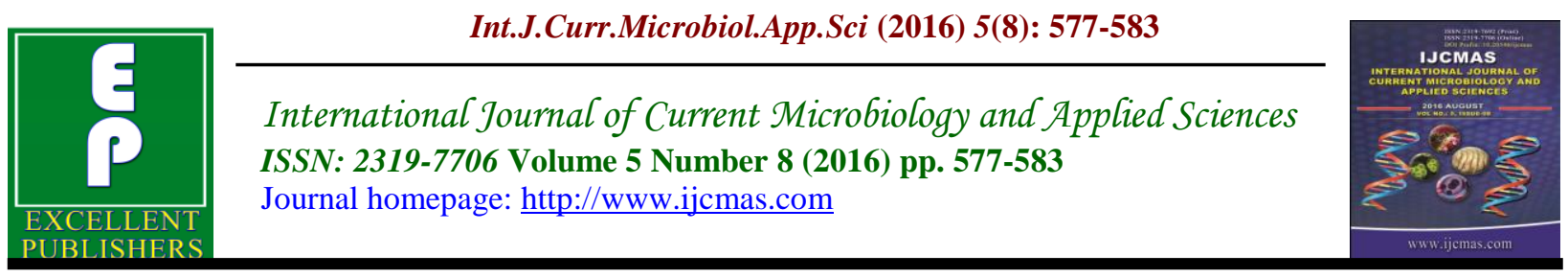

Original Research Article

http://dx.doi.org/10.20546/ijcmas.2016.508.064

\title{
Spectrum Actigraphy Sleep and Caregiver Wellbeing
}

\author{
Greg Clare*, I.J. Washburn and P. Hebert \\ Design, Housing and Merchandising Oklahoma State University 431 Human Sciences \\ Stillwater, OK 74078, U.S.A. \\ *Corresponding author
}

\begin{abstract}
A B S T R A C T
Keywords

Circadian rhythms,

Sleep Behaviors,

Lighting,

Actigraph

Spectrum.

Article Info

Accepted:

24 July 2016

Available Online:

10 August 2016

Healthy adult shift workers from continuing care retirement centers wore Philip's Actigraph Spectrum watches for a 21-day period to generate pilot data about the effects of natural and built environmental light exposures on sleep behaviors. The study found that average levels of light are more important to prediction of the behavioral and sleep for predicting the outcomes than the maximum levels. However, for blue light, only the maximum levels predicted the outcomes. Dummy variables measuring the environmental light model effects on sleep including caregiver work shifts (morning, afternoon, night) did not significantly influence sleep behaviors, however self-reported work and family stress demonstrated significant effects on sleep variables while financial stress did not among the caregiver group.
\end{abstract}

\section{Introduction}

There is a critical need to better understand the circadian rhythms of caregivers in older adult continuing care retirement centers (CCRC). Projected demographic changes globally of persons over 65 years highlight the need of a healthy caregiver workforce to address emerging population increases of older adults (Guseh, 2016). Circadian rhythms are 24-hour body clock cycles which are important for human health and are influenced by light exposure, physical activity and other factors. Light has been demonstrated to influence human health through the periodic exposure to light sources over a 24-hour period known as the circadian rhythm cycle.
Lightness and darkness influence gene expression, which in turn regulates human biological systems as part of the circadian rhythm cycle (Stevens \& Rea, 2001; Davis \& Mirick, 2006). Research suggests that shiftwork may be related to adverse health effects and these risks increase with the age of the worker (Akerstedt, 2003; Drake et al., 2004). There is a need to better understand how specific work environments such as CCRCs with varied exposure to natural light during work shifts, may influence these risks (Ramin et al., 2014).

Drifting or disrupted circadian rhythms create irregular sleep/wakefulness patterns 
which are sometimes observed in those who work night or early morning shifts; are of advanced age; or have disorders such as Alzheimer's. The goal of this pilot study was to generate data that measured the effects of visible light exposures including the red, green, and blue spectral ranges on worker sleep performance at three large continuing care retirement centers in Oklahoma. Workers are normally exposed to a combination of natural and artificial light sources over a 24-hour period known as the circadian rhythm cycle. Differential exposure to alternative forms of natural and artificial light such as levels that are experienced by shift workers has been demonstrated to play a critical role in circadian rhythm disruption (Stevens et al., 2007; Stevens \& Zhu, 2015). Sleep disruption is generally believed to be synonymous with circadian rhythm disruption (Gottlieb, O'Connor \& Wilk, 2007).

Circadian rhythm disruption is increasingly recognized as a source of substantial morbidity; chronic illnesses such as depression, cancer, anxiety, obesity, and heart disease may be influenced by circadian rhythm disruption. (Sephton \& Spiegel, 2003; Pauley, 2004; Davis \& Mirick, 2006; Schernhammer et al., 2006; Buxton et al., 2012; Zhu \& Zee, 2012; Haus \& Smolensky, 2013).Shiftworkers employed with rapidly rotating and overnight shifts may be at risk of developing imbalanced circadian rhythm cycles from limited exposure to natural light during night shifts and sleep during daylight hours. Continuing care retirement centers may also contribute to disrupted sleep due to work and home stress factors such as: long shifts, urgency and complexity of work duties, fatigue, limited staffing, family and financial stress (Perron \& Friedlander, 1996; Whitmire et al., 2009). Access to special populations in field settings such as continuing care retirement centers is vital to our understanding the effects of visible light on human health. Research suggests that modifications of light in the narrow bandwidth of 211 to $405 \mathrm{~nm}$ produce reductions in melatonin production in healthy human subjects that directly influences the sleep cycle (Brainard et al., 2013). CCRC workers may exhibit differing patterns of alertness and fatigue than office workers due to assigned work duties. This study adds new knowledge to the body of circadian rhythm and comparative workplace literature for CCRC.

\section{Methods}

The researchers posit that creating resilient caregivers requires a balance of mental and physical activity and rest both at work and outside of work. Accessing groups of caregivers who experience varying degrees of work and home-life pressures and collectively comparing environmental light exposures to sleep performance data will offer preliminary insights into specialized workforces at CCRC. To accomplish this goal, the researchers used Philip's spectrum actigraphy watches. Actigraphy watches measure variations to sleep patterns and quality over time in the presence of environmental light exposures.

Seventy-five CCRC shiftworkers were recruited for the study from 3 facilities. Characteristics of the sample may be found in Table 1. Recruitment was completed in partnership with facility management and participants were compensated $\$ 100$ for participating in the study. After completing informed consent documentation, participants answered a paper survey and agreed to wear a Philip's Actigraph Spectrum PRO watch for a period of 21 days, 24 hours a day. Recruited participants worked at the facilities in a combination of 
day, afternoon, evening, or rapidly rotating shifts. Participants' were asked on the survey to report the frequency with which they feel work, family, and financial stress. Each subject's identity was protected during the study through assignment of a subject number associated with their actigraphy watch (e.g. 1-75).

The research team performed weekly touchbase visits to the CCRC's and consulted with management and the participants on the successful progress of actigraphy watch functionality and data collection during the 21-day period. The research team reacted to any equipment issues and participant concerns during the facility visits. One of the subjects actigraphy data was removed from the sample due to technical issues with the actigraphy watch and no data collected $(n=74)$.

At the completion of the 21-day period, the watches were downloaded at the partnering CCRC facilities. After download, participants watched an actigraphy methods debriefing video lasting approximately 10 minutes and a copy of a personalized Philip's Actiware clinician's report. The actigraphy report recaps lighting exposures, accelerometer based activity levels and sleep performance metrics during the 21-day collection period. The sleep performance variables of interest for the present study included: activity, wake, sleep, and immobility measures. In addition to the actigraphy report, participants received a copy of the CDC sleep hygiene fact sheet for the state of Oklahoma to increase knowledge of the importance of adequate sleep.

\section{Analysis}

Given the clustered nature of the data (1205 observations from 74 people) the researchers ran multilevel regression in STATA 14 statistical software with random intercepts for the eight outcomes. Each outcome was run twice, once with the maximum light variables and one with average light variables (both transformed using a natural logarithm). A follow-up analysis introduced dummy variables to the panel data multilevel regression analysis with random intercepts which measured shifts worked at the facility (i.e. morning, afternoon, and evening) and self-reported stress levels from the paper survey.

\section{Results and Discussion}

The results for all outcomes and both models are presented in Table 2. Generally, across outcomes, the average levels of light are more important to prediction of the outcomes than the maximum levels. However, for blue light, only the maximum levels predicted the outcomes. Varied caregiver shift assignments did not influence sleep behaviors for average or maximum light exposure levels. Self-reported work stress levels demonstrated a minor fixed significant effects in average light level for maximum activity in sleep time and immobile time. Similar minor fixed effects were demonstrated for maximum light levels for sleep time and immobile time. The data also suggests that as family stress decreases maximum activity count in both maximum and average lighting conditions.

Low-cost and widely deployable lighting interventions to improve shift worker health may support entrainment of the circadian clock and potentially influence health risk indicators for at risk employees. Ways in the workplaces to reduce stress supported by circadian lighting systems may offer one solution to improving worker perceived well-being while at work. In the near future, emerging low-cost spectrum actigraphy devices may be wirelessly linked to network 
controlled task lights and bio-metric monitoring systems to modify lighting levels and spectral profiles on-demand, thereby impacting circadian rhythm performance and improving human health on a per worker basis. However, substantial research in this evolving area of workplace monitoring and entrainment is needed in both laboratory and field settings.

Table.1 Characteristics of the Sample

\begin{tabular}{|c|c|c|}
\hline & $\#$ & $\%$ \\
\hline \multicolumn{3}{|l|}{ Gender } \\
\hline Male & 11 & $15 \%$ \\
\hline Female & 64 & $85 \%$ \\
\hline Total & 75 & $100 \%$ \\
\hline \multicolumn{3}{|l|}{ Race/Ethnicity (Multi-Report) } \\
\hline White & 58 & $73 \%$ \\
\hline African American & 7 & $9 \%$ \\
\hline Asian & 1 & $1 \%$ \\
\hline Native American & 13 & $16 \%$ \\
\hline Total & 79 & $100 \%$ \\
\hline \multicolumn{3}{|l|}{ Education } \\
\hline Less than High School & 3 & $4 \%$ \\
\hline High School/GED & 25 & $33 \%$ \\
\hline Some College & 29 & $39 \%$ \\
\hline 2-Year College Degree & 6 & $8 \%$ \\
\hline 4-Year College Degree & 8 & $11 \%$ \\
\hline \multirow[t]{2}{*}{ Master's Degree } & 4 & $5 \%$ \\
\hline & 75 & $100 \%$ \\
\hline \multicolumn{3}{|l|}{ Income } \\
\hline Below $\$ 20,000$ & 19 & $26 \%$ \\
\hline$\$ 20,000-\$ 39,999$ & 35 & $49 \%$ \\
\hline$\$ 40,000-\$ 69,999$ & 5 & $7 \%$ \\
\hline$\$ 70,000-\$ 89,999$ & 10 & $14 \%$ \\
\hline \multirow[t]{2}{*}{$\$ 90,000$ or more } & 3 & $4 \%$ \\
\hline & 72 & $100 \%$ \\
\hline
\end{tabular}


Table.2 Results of Light Spectrums on Behavioral Measures

\begin{tabular}{|c|c|c|c|c|c|c|c|c|c|c|c|c|c|c|c|c|}
\hline \multirow[b]{3}{*}{ Avg. White } & \multirow{2}{*}{\multicolumn{2}{|c|}{$\begin{array}{c}\text { Total } \\
\text { AC }\end{array}$}} & \multirow{2}{*}{\multicolumn{2}{|c|}{$\begin{array}{c}\text { Max } \\
\text { AC }\end{array}$}} & \multirow{2}{*}{\multicolumn{2}{|c|}{$\begin{array}{l}\text { Wake } \\
\text { Time }\end{array}$}} & \multirow{2}{*}{\multicolumn{2}{|c|}{ Wake }} & \multirow{2}{*}{\multicolumn{2}{|c|}{$\begin{array}{c}\text { Sleep } \\
\text { Time }\end{array}$}} & \multirow{2}{*}{\multicolumn{2}{|c|}{ Sleep }} & \multirow{2}{*}{\multicolumn{2}{|c|}{$\begin{array}{c}\text { Immobile } \\
\text { Time }\end{array}$}} & \multirow{2}{*}{\multicolumn{2}{|c|}{ Immobile }} \\
\hline & & & & & & & & & & & & & & & & \\
\hline & -0.13 & $* *$ & -0.07 & $* *$ & $-0.06 *$ & & -0.03 & & -0.01 & & -0.04 & & -0.01 & & -0.04 & $*$ \\
\hline Avg. Red & 0.18 & $* * *$ & 0.07 & $* * *$ & $0.11 *$ & $* * *$ & $0.07 *$ & $* * *$ & $0.01 \dagger$ & & 0.07 & $* * *$ & 0.01 & $\dagger$ & 0.06 & $* * *$ \\
\hline Avg. Green & 0.11 & $* * *$ & $0.05^{\prime}$ & $* * *$ & $0.07 *$ & $* * *$ & $0.04 *$ & $* *$ & $0.01 *$ & & 0.04 & $* *$ & 0.01 & $*$ & 0.03 & $* *$ \\
\hline Avg. Blue & 0.01 & & 0 & & -0.002 & & 0 & & 0 & & 0 & & 0 & & 0 & \\
\hline Morning Shift & -0.07 & & -0.03 & & -0.04 & & -0 & & -0.02 & & 0 & & -0.02 & & -0.02 & \\
\hline Afternoon Shift & 0.08 & & 0 & & 0.03 & & 0.01 & & 0.01 & & 0.01 & & 0.02 & & -0.06 & \\
\hline Work Stress & 0.13 & & 0.042 & & 0.10 & & 0.02 & & $0.04 *$ & & 0.02 & & 0.04 & $*$ & 0.04 & \\
\hline Financial Stress & 0.08 & & 0.092 & & 0.10 & & 0.12 & & -0.02 & & 0.12 & & -0.03 & & 0.1 & \\
\hline Family Stress & -0.07 & & -0.1 & & -0.05 & & -0.09 & & 0.02 & & -0.09 & & 0.03 & & -0.08 & \\
\hline Intercept & 9.65 & $* * *$ & $7.01=$ & $* * *$ & $4.6 *$ & $* * *$ & & $* * *$ & $6.97 *$ & $* * *$ & 3 ; & $* * *$ & $6.85 *$ & $* * *$ & 3.22 & $* * *$ \\
\hline Max White & -0.09 & & -0.09 & $* *$ & -0.05 & & -0.04 & & 0 & & -0.05 & $\dagger$ & 0 & & -0.03 & \\
\hline Max Red & 0.14 & $* * *$ & 0.09 & $* * *$ & 0.08 * & $* * *$ & 0.06 * & $* * *$ & 0.01 & & 0.05 & $* * *$ & 0.01 & & 0.04 & $* *$ \\
\hline Max Green & 0.07 & $*$ & $0.05=$ & $*$ & $0.05 \dagger$ & $\dagger$ & $0.03 *$ & & 0 & & 0.05 * & $* *$ & 0 & & 0.03 & * \\
\hline Max Blue & 0.03 & $*$ & $0.02=$ & $*$ & 0.01 & & 0 & & $0.01 *$ & & 0 & & 0.01 & $*$ & 0 & \\
\hline Morning Shift & -0.08 & & -0.04 & & -0.04 & & -0.01 & & -0.02 & & 0.01 & & -0.02 & & -0.02 & \\
\hline Afternoon Shift & 0.03 & & -0.03 & & 0.01 & & -0.01 & & 0.00 & & 0.07 & & 0.01 & & -0.07 & \\
\hline Work Stress & 0.12 & & 0.04 & & 0.10 & & 0.02 & & $0.04 *$ & & 0.02 & & 0.04 & $*$ & 0.03 & \\
\hline Financial Stress & 0.13 & & 0.11 & & 0.13 & & $0.15 \dagger$ & & -0.02 & & 0.15 & $\dagger$ & -0.02 & & 0.12 & \\
\hline Family Stress & -0.12 & & -0.12 & & 0.08 & & 0.11 & & 0.02 & & -0.11 & & 0.03 & & -0.1 & \\
\hline Intercept & 9.1 & $* * *$ & $6.9=$ & $* * *$ & $4.3 *$ & $* * *$ & $2.9 *$ & $* * *$ & $7.0 *$ & $* * *$ & $2.9^{*}$ & $* * *$ & $6.8^{*}$ & $* * *$ & 3.1 & * \\
\hline
\end{tabular}

$* * * \mathrm{p}<.001, * * \mathbf{p}<.01, * \mathbf{p}<.05, \dagger \mathbf{p}<.10$

The long-term goal of this research agenda is to determine if ergonomic lighting interventions used in combination with actigraphy device monitoring and real-time network controlled light-levels impact the sleep cycle and reduce the risk of disease for large groups of in situ shift workers over time. Substantial laboratory research has proposed that the circadian clock may be regulated by lighting interventions (Yoon, $e t$ al 2002; Boivin and James, 2002; Figueiro and Rea, 2006, 2010; Grundy et al., 2009; Pallesen et al, 2010; Kantermann \& Roenneberg, 2009;Sahin et al., 2013, Sahin\& Figueiro, 2014; Guo et al., 2014). Field research in varied industry sectors amongthe narrow bandwidth of 211 to 405 $n m$ produce reductions in melatonin production in healthy human subjects that directly influences the sleep cycle (Brainard et al., 2013). CCRC workers may exhibit differing patterns of alertness and fatigue than workers in other sectors due to assigned their work duties and mitigated by nonworkplace stress factors. Studies of multiple shiftwork sectors and employee groups is needed. In addition, researchers need to better understand the relationship between perceived worker stress and myriad worker light exposures (i.e. natural and artificial) during the 24-hour circadian cycle. The goal of reducing worker stress, increasing productivity and sleep performance may be related to lighting exposures as suggested by the findings of this study. Spiraling healthcare costs and the need for a healthy workforce to meet the needs of an aging population highlight the need for additional 
research using spectrum actigraphy in diverse field workplace settings. The next stage of this research agenda will measure the effects of lighting interventions and realtime measurement of self-reported stress.

\section{References}

Åkerstedt, T. 2003. Shift work and disturbed sleep/wakefulness. Occupational Medi., 53(2): 89-94.

Boivin, D.B., and James, F.O. 2002. Circadian adaptation to night-shift work by judicious light and darkness exposure. J. Biol. Rhythms, 17: 556567.

Brainard, G.C., Coyle, W., Ayers, M., Kemp, J., Warfield, B., Maida, J., \& Hanifin, J.P. 2013. Solid-state lighting for the international space station: tests of visual performance and melatonin regulation. Acta Astronautica,92(1): 21-28.

Buxton, O.M., Cain, S.W., O’Connor, S.P., Porter, J.H., Duffy, J.F., Wang, W., \& Shea, S.A. 2012. Adverse metabolic consequences in humans of prolonged sleep restriction combined with circadian disruption. Sci. Translational Medi., 4(129): 129ra43-129ra43.

Davis, S., Mirick, D.K. 2006. Circadian disruption, shift work and the risk of cancer: a summary of the evidence and studies in Seattle. Cancer Causes \& Control, 17(4): 539-545.

Drake, C.L., Roehrs, T., Richardson, G., Walsh, J.K., \& Roth, T. 2004. Shift work sleep disorder: prevalence and consequences beyond that of symptomatic day workers. Sleep, 27(8): 1453-1462.

Figueiro, M.G., \& Rea, M.S. 2010. The effects of red and blue lights on circadian variations in cortisol, alpha amylase, and melatonin. Int. J. Endocrinol.

Figueiro, M.G., Rea, M.S., \& Bullough, J.D.
2006. Does architectural lighting contribute to breast cancer? $J$. Carcinogenesis, 5(1): 20.

Gottlieb, D.J., T O'Connor, G., \& Wilk, J.B. 2007. Genome-wide association of sleep and circadian phenotypes. BMC medical genetics, 8 (Supplement 1).

Grundy, A., Sanchez, M., Richardson, H., Tranmer, J., Borugian, M., Graham, C. H., \& Aronson, K.J. 2009. Light intensity exposure, sleep duration, physical activity, and biomarkers of melatonin among rotating shift nurses, Chronobiol. Int., 26(7): 1443-1461.

Guo, J.H., Qu, W.M., Chen, S.G., Chen, X.P., Lv, K., Huang, Z.L., \& Wu, Y.L. 2014. Keeping the right time in space: importance of circadian clock and sleep for physiology and performance of astronauts. Military Medi. Res., 1(1): 23.

Guseh, J.S. 2016. Aging of the World's Population. The Wiley Blackwell Encyclopedia of Family Studies.

Haus, E.L., \& Smolensky, M.H. 2013. Shift work and cancer risk: potential mechanistic roles of circadian disruption, light at night, and sleep deprivation, Sleep Medi. Rev., 17(4): 273-284.

Kantermann, T., Roenneberg, T. 2009. Is light-at-night a health risk factor or a health risk predictor? Chronobiol. Int., 26(6): 1069-1074.

Pallesen, S., Bjorvatn, B., Magerøy, N., Saksvik, I.B., Waage, S., Moen, B.E. 2010. Measures to counteract the negative effects of night work, Scandinavian J. Work, Environ. Health, 109-120.

Pauley, S.M. 2004. Lighting for the human circadian clock: recent research indicates that lighting has become a public health issue. Medical Hypotheses, 63(4): 588-596.

Perron, M.J., Friedlander, R.H. 1996. The 
effects of downsizing on safety in the CPI/HPI. Process safety progress, 15(1), 18-25.

Ramin, C., Devore, E. E., Wang, W., PierrePaul, J., Wegrzyn, L. R., \& Schernhammer, E. S. 2014. Night shift work at specific age ranges and chronic disease risk factors. Occupational and environmental medicine, oemed-2014.

Sahin, L., Wood, B.M., Plitnick, B., \& Figueiro, M.G. 2014. Daytime light exposure: Effects on biomarkers, measures of alertness, and performance, Behavioral Brain Res., 274: 176-185.

Sahin, L., \& Figueiro, M.G. 2013. Alerting effects of short-wavelength (blue) and long-wavelength (red) lights in the afternoon. Physiol. Behavior, 116: 1-7.

Santhi, N., Thorne, H.C., van der Veen, D. R., Johnsen, S., Mills, S. L., Hommes, V., Dijk, D.J. 2012. The spectral composition of evening light and individual differences in the suppression of melatonin and delay of sleep in humans. J. Pineal Res., 53(1): 47-59.

Schernhammer, E.S., Kroenke, C.H., Laden, F., \& Hankinson, S.E. 2006. Night work and risk of breast cancer. Epidemiol., 17(1): 108-111.

Sephton, S., Spiegel, D. 2003. Circadian disruption in cancer: a neuroendocrine-immune pathway from stress to disease? Brain, Behavior Immunity, 17(5): 321-328.

Stevens, R.G., Blask, D.E., Brainard, G.C.,
Hansen, J., Lockley, S., Provencio, I., Rea, M.S., Reinlib, L. 2007. Meeting Report: The role of environmental lighting and circadian disruption in cancer and other diseases. Environmental Health Perspectives, 115: 1357-1362.

Stevens, R.G., \& Rea, M.S. 2001. Light in the built environment: potential role of circadian disruption in endocrine disruption and breast cancer. Cancer Causes \& Control, 12(3), 279-287.

Stevens, R. G., \& Zhu, Y.2015. Electric light, particularly at night, disrupts human circadian rhythmicity: is that a problem? Philosophical transactions of the Royal Society of London B: Biol. Sci., 70(1667), 20140120.

Whitmire, A., Leveton, L.B., Barger, L., Brainard, G., Dinges, D.F., Klerman, E., Shea, C. 2009. Risk of performance errors due to sleep loss, circadian desynchronization, fatigue, and work overload. Evidence-based review by NASA Behavioral Health and Performance Program, Johnson Space Center, 85-117.

Yoon, I.Y., Jeong, D.U., Kwon, K.B., Kang, S.B., and Song, B.G. 2002. Bright light exposure at night and light attenuation in the morning improve adaptation of night shift workers. Sleep, 25: 351-356.

Zhu, L., \& Zee, P.C. 2012. Circadian Rhythm Sleep Disorders. Neurologic Clinics, 30(4): 1167-1191. doi:10.1016/j.ncl.2012.08.011

\section{How to cite this article:}

Greg Clare, I.J. Washburn and P. Hebert. 2016. Spectrum Actigraphy Sleep and Caregiver Wellbeing. Int.J.Curr.Microbiol.App.Sci. 5(8): 577-583. doi: http://dx.doi.org/10.20546/ijcmas.2016.508.064 